\title{
Cosmic rays and the structure of the universe studied in Cosmic Ray Extremely Distributed Observatory with citizen science
}

\author{
Robert Kamiński, ${ }^{a, b, *}$ Janusz Firla ${ }^{b}$ and Sławomir Stuglik ${ }^{a, b, *}$ on behalf of the \\ CREDO Collaboration \\ (a complete list of authors can be found at the end of the proceedings) \\ a Institute of Nuclear Physics Polish Academy of Sciences, \\ Radzikowskiego 152, Cracow, Poland \\ ${ }^{b}$ CREDO project \\ E-mail: robert.kaminski@ifj.edu.pl, kragpokolen@wp.pl, \\ slawomir.stuglik@ifj.edu.pl
}

The CREDO project aims to observe sets of simultaneous global air showers, i.e. covering the entire hemisphere of the Earth. To do this, it is necessary to deploy detectors of various sizes as densely as possible across the globe. These observations are intended to help answer some basic questions of astrophysics, such as the occurrence and nature of dark matter, the sources of high-energy particles, and the structure of space-time. The method of work of the CREDO project is citizen science, i.e. participation in the project activities not only of scientists but also students, adults, organized groups of enthusiasts and individuals interested in the topic. This community also includes people with disabilities, e.g. visually impaired or blind. The synergy of these two tasks of CREDO - science and education of the society, results in an increasing and wider territorial participation of outsiders in the activities of the project and a very rapid scientific development of the project. The following text presents two aspects of the CREDO project scientific and educational, and the state of art of the project for June 2021.

$37^{\text {th }}$ International Cosmic Ray Conference (ICRC 2021)

July 12th-23rd, 2021

Online - Berlin, Germany

\footnotetext{
*Presenter
} 


\section{Introduction}

CREDO, or Cosmic Ray Extremely Distributed Observatory, is a scientific project created at Institute of Nuclear Physics Polish Academy of Sciences in Cracow (hereafter INP PAS) in 2016 and is currently dynamically developing around the world. His innovation is a very large increase in the range of simultaneous measurements of air showers to the entire Earth and the involvement of all people from outside the group of scientists [1]. CREDO is primarily a research project and popularization in the spirit of citizen science is (which is very important) an integral part of this project without which the project simply could not work. One can even say that it is a condition for the existence of the CREDO project and the interpenetration of these two aspects (science and popularization (or education)) brings very good scientific and didactic results. Popularization is not an aspect added to the project, it is the principle and method of its existence, from the beginning of the project. Therefore, CREDO operates in two areas: science and popularization of science (education) at the same time showing how important this connection is for achieving current and distant goals in time. Not only scientific (current and future) achievements are important here, but also sociological achievements related to the continuation of these and other astrophysical research in the future. In the text below, both the scientific research and goals as well as the popularization/educational activity of CREDO will be briefly presented.

\section{Science in CREDO project}

The Fig. 1 shows the appearance of a typical single air shower. Its diameter when hitting the Earth's surface is about $1 \mathrm{~km}$. It is a single tuft and can be counted on to be registered by detectors placed locally at the point (area) where the tuft hits. However, if we are dealing with a whole set of thousands or millions of such showers hitting the entire hemisphere of the Earth at the same time, then such a local set of detectors is not enough. The Fig. 1 shows a schematic of such a set of atmospheric showers. One needs a whole network of detectors spread out as densely as possible and in the largest possible area. CREDO aims to organize such a network all over the world.

The source of such sets of air showers may be, for example, decays of very massive, still unknown but desired particles, e.g. dark matter. A theoretically possible diagram of such decay of such a particle and the production of super pre shower is shown in the Fig. 2. In this way, CREDO has the potential to study the structure of the universe on a micro scale, i.e. on the scale of elementary particles. These studies may go beyond the Standard Model which is, after confirming the existence of the Higgs boson and thus completing list of intermediary bosons in the Standard Model, a much needed and recently hot topic of physicists' research. Such research will be increasingly needed over the coming decades and will focus the attention of many physicists and astrophysicists.

The second reason for the necessity to expand the base for the distribution of detectors to the entire Earth are the observations of particles with very high energy - even above $10^{20} \mathrm{eV}$, which CREDO wants to implement. A very small number of these particles $\left(1 / \mathrm{km}^{2} /\right.$ years $)$ compared to those with less energy requires:

1. increase the observation time to several hundred or several thousand years with a small network of detectors or

2. a significant increase in the detector distribution area. 

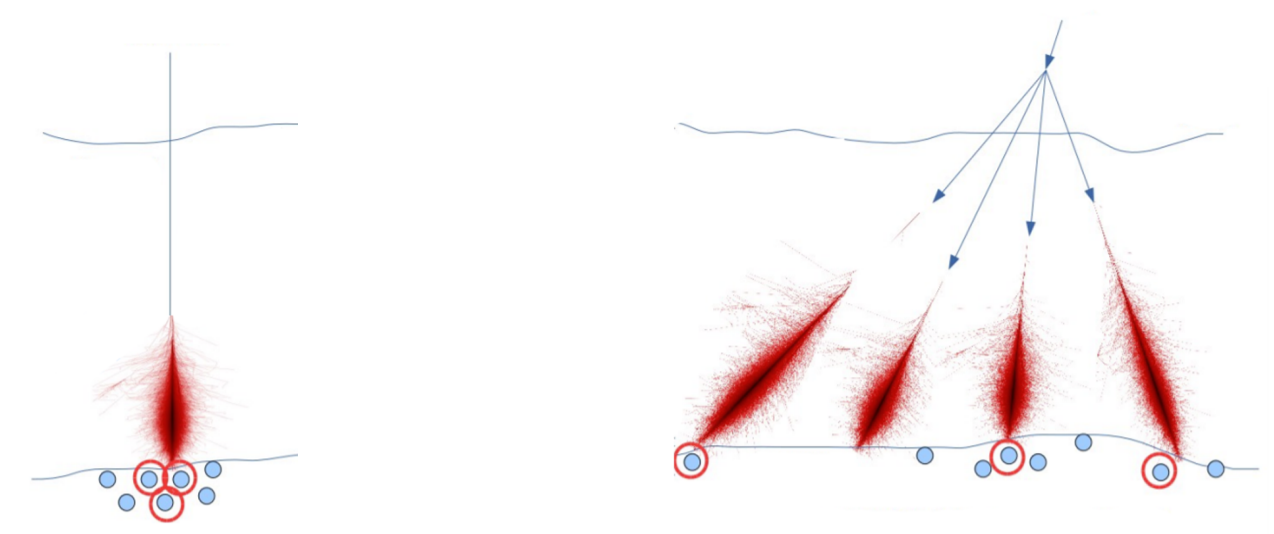

Figure 1: Left - single air shower, right - a bunch of simultaneous air showers
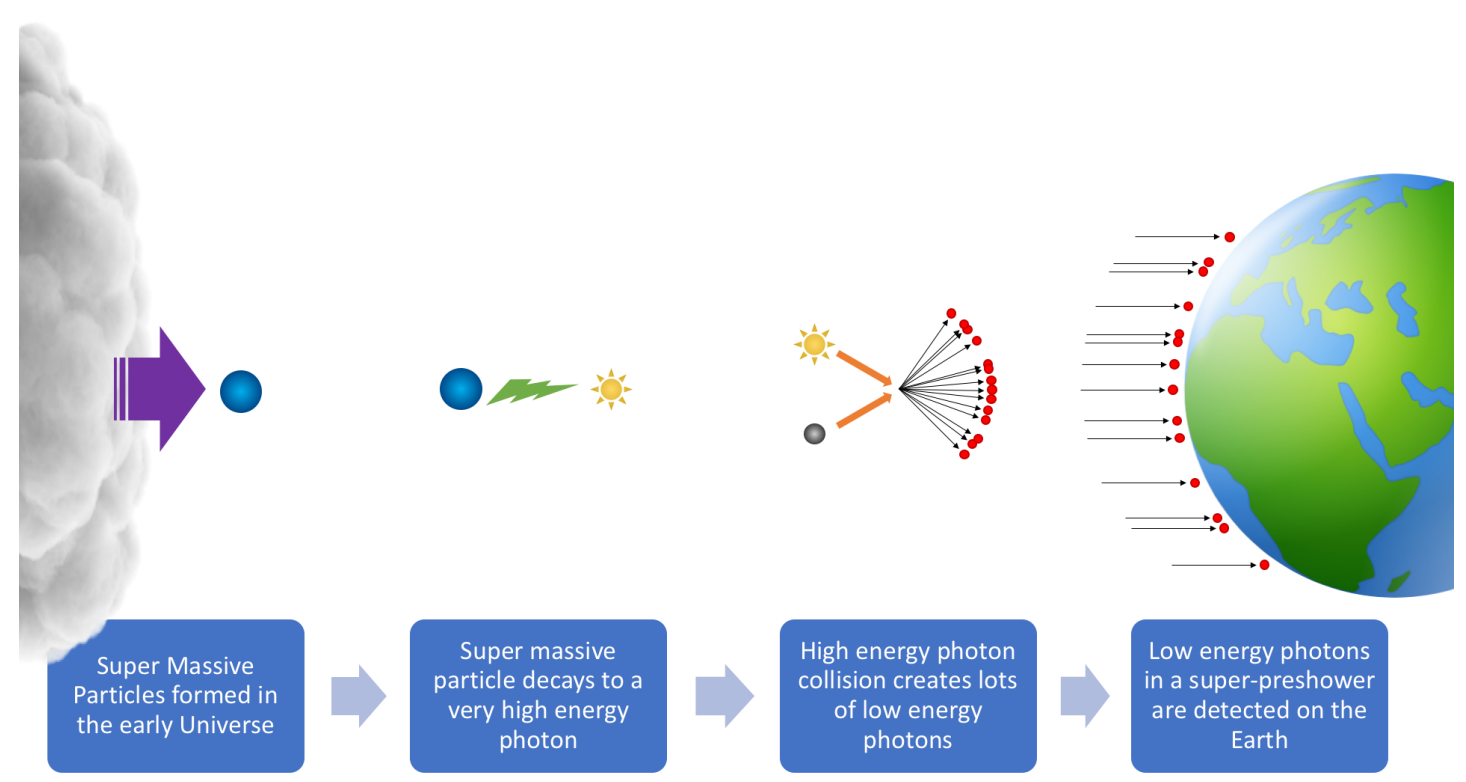

Low energy photons

in a super-preshower

are detected on the

$$
\text { Earth }
$$

Figure 2: Diagram of the possible decay of a hypothetical heavy particle

The assumptions of the CREDO operation from the very beginning in $100 \%$ meet the second condition, which can put it at the forefront of projects with such a scientific goal. The importance of research on such high-energy particles relates to the analysis of the structure of the universe, i.e. research on a macro scale. It is precisely particles with such high energies, i.e. very short wavelengths, that can be sensitive to very small inhomogeneities in the structure of space-time, unlike particles with lower energy, i.e. longer wavelengths. These differences may cause, for example, a delay in the arrival of high-energy particles (e.g. from a supernova explosion) due to 
the interaction of very short waves with very small inhomogeneities in the space-time structure.

In addition to these strictly astrophysical aspects of CREDO's activity, at least one more needs to be mentioned (for the time being at the stage of development): researching the impact of disturbances in the magnetic field before earthquakes on the number of recorded cosmic particles. If the close correlations between these phenomena are confirmed, it will be possible to use the network of detectors organized by CREDO to detect signs of an impending earthquake (a few hours before) and alert the population at risk.

It is also worth mentioning that the cosmic rays studied by CREDO, also created by photons of different energies, may be of significant importance for the study of photoproduction of e.g. mesons or exotic states within the Standard Model and beyond it. Such studies are very important for a better understanding of the Standard Model and for the search for resonances deviating from the usual - two and three quark - structures. These tests can help in the works carried out in ground - specially designed for this purpose laboratories, e.g. in JLab (Clas12 and GlueX detectors).

\section{Science and popularization in CREDO project}

Another important aspect of promoting the CREDO project is its low cost. Currently, the project uses small but widely available detectors in smartphones. Registration of cosmic particles only requires installing free applications created by CREDO for Android and IoS and using them as recommended. Collecting even huge amounts of data collected by camera matrices in smartphones at the Cyfronet Institute in Cracow is also cheap, so it is possible for people from outside of science to participate from the very beginning - i.e. from data collection. An example of data appearance is shown in the Fig. 3. It can be seen that it is necessary from the very beginning identify (i.e. examine the origin) of the traces, then perform their initial analysis and finally joint analysis with scientists from CREDO. The diagram of the path of such an analysis is presented in the Fig. 4. Its implementation is possible thanks to the principle of availability of all data to all interested parties, adopted by citizen science.

In order for all the described stages - from collecting data through the analysis of traces in matrices to the development of final specific scientific results - to be possible, CREDO from the very beginning intensifies popularization activities among school students, teachers and other interested parties. Table 1 presents information on the popularization activities carried out and organized by CREDO. There is a clear and continuous increase in their number (except for 2020 for epidemiological reasons) and this trend CREDO wants to maintain and strengthen.

The events listed in this table were individual and apart from them, CREDO also runs long-term activities, which include: annual Particle Hunter competitions (several dozen schools take part in them) and summer two-month competitions for all interested parties. It is important for CREDO to work on including in the detection of particle traces and their analysis of people with disabilities, e.g. visually impaired or blind. It is based on the so-called sonification of registration and analysis of traces and started in year 2021 or more generally - on typhlographics - processing of depictions of basic knowledge about the cosmos into tactile forms. These works were largely inspired by the astrophysicist Wanda Diaz Merced, who also advocates imaging gravitational waves in order to provide the latest and numerous (in the coming years) data from observatories such as LIGO, VIRGO and others for the visually impaired. To learn more about these ideas, please see e.g. [2]. 

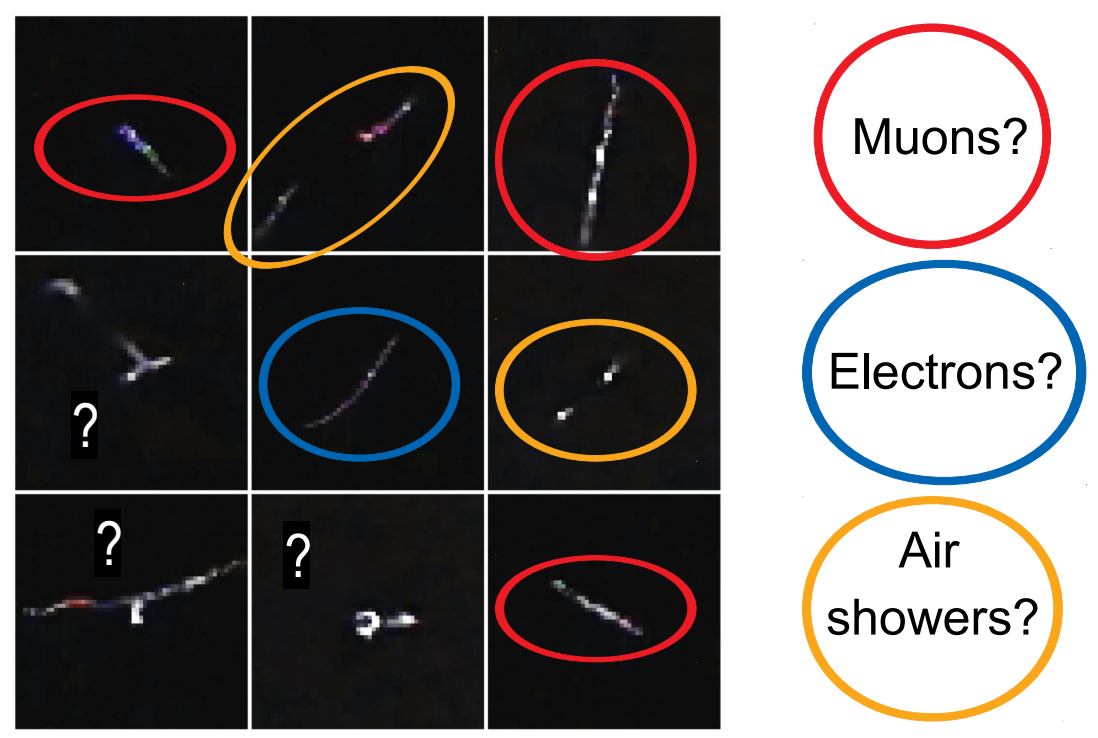

Figure 3: Examples of registered traces of particles in smartphone camera matrices with possible identification. The sign "?" marks the traces that are most difficult to identify.

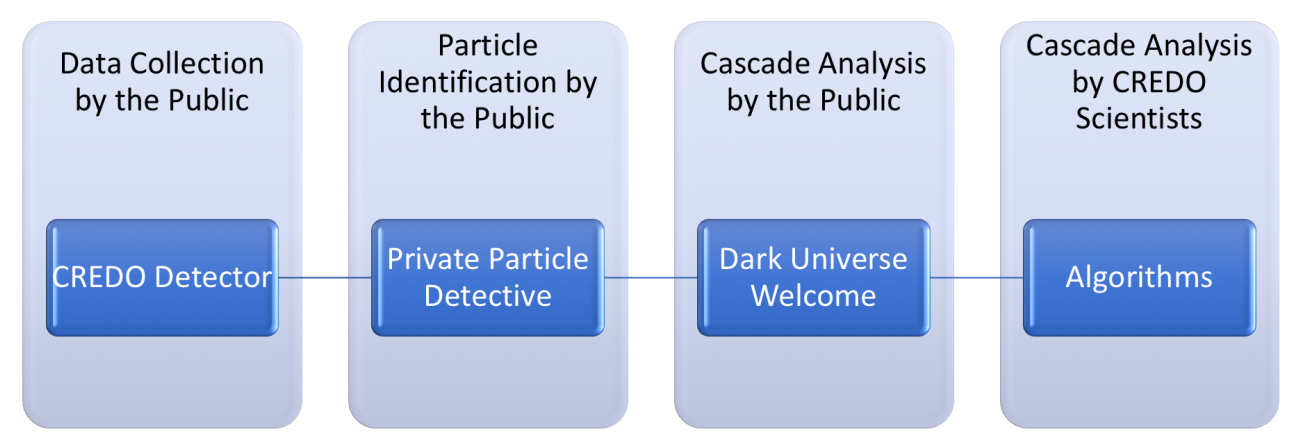

Figure 4: Scheme of analysis from trace recording to final scientific analysis

Important is to stress here again that popularization is not an aspect added to the project, it is the principle and method of its existence, from the beginning of the project.

Fig. 5 concerns the aspect of popularizing CREDO and shows a map of application users for the month of June year 2021. As one can see there are several thousand users in total (still most of them in Europe), dozens of scientific institutions around the world cooperate with CREDO and several million traces of particles are collected. The number of collected data gives a great chance for a reliable analysis carried out under the supervision of CREDO academics and requires the involvement of many people 
Table 1: Events or attendance organized by CREDO. In columns are successively: year, name and place

\begin{tabular}{|c|c|c|}
\hline 2016 & CREDO Inauguration & INP PAS \\
\hline \multirow{4}{*}{2017} & International Cosmic Day 2017 & INP PAS \\
\hline \multirow{4}{*}{2018} & CREDO Anniversary Symposium & INP PAS \\
& CREDO Visegrad Project Meeting & Bratislava \\
& CREDO in school & Kraków \\
& CREDO Week & INP PAS \\
& Scientists' Night & INP PAS \\
& science picnic & Kraków \\
& meetings in schools & Poland \\
& School meeting & Siemianowice \\
\hline \multirow{7}{*}{2019} & CREDO Workshop & INP PAS \\
& Visegrad Workshop & Opava \\
& Scientists' Night & INP PAS \\
& science picnic & Kraków \\
& science picnic & Olsztyn \\
& science picnic & Warszawa \\
& meetings in schools & Poland \\
\hline \multirow{3}{*}{2020} & Scientists' Night & INP PAS \\
& Open Day & INP PAS \\
\hline & Scientists' Night & INP PAS \\
& EduSky conference for teachers & Kraków \\
& Physics as the key to understanding the world- & \\
& -CREDO and cosmic rays & INP PAS \\
& Open Day & INP PAS \\
\hline
\end{tabular}

\section{Conclusions}

The CREDO project has a chance to join the group of the most important scientifically recognized cosmic particle registration projects in the world. The analyzes performed as part of its activities can be unique on a global scale and shed new light on new results and new methods. It is the citizen science methods used by CREDO that give it a special place in the pantheon of the most scientifically and sociologically useful research projects in the world. The network of detectors based on cameras in smartphones used and disseminated by CREDO is only the first step towards achieving the goal of universal, global network of detectors around the world. Further steps are the development of networks of larger and more accurate detectors and the continuous education of social groups around the world in order to enable more efficient detection and increase the quality of analysis of the collected data as part of the CREDO - citizen science method.

The number of publications and citations for such a young project seems impressive. These numbers are: (year/number of publications/number of citations) 2017/3/17, 2018/6/23, 2019/8/12, 2020/8/15 and 2021/4/5. To find links to many CREDO works, see the bibliographies in [3]. The number of people very actively involved in work for CREDO is also noteworthy - about 30 people (summer 2021), including apprentices, graduate students, doctoral students, researchers from INP 


\section{CREDO: already global}

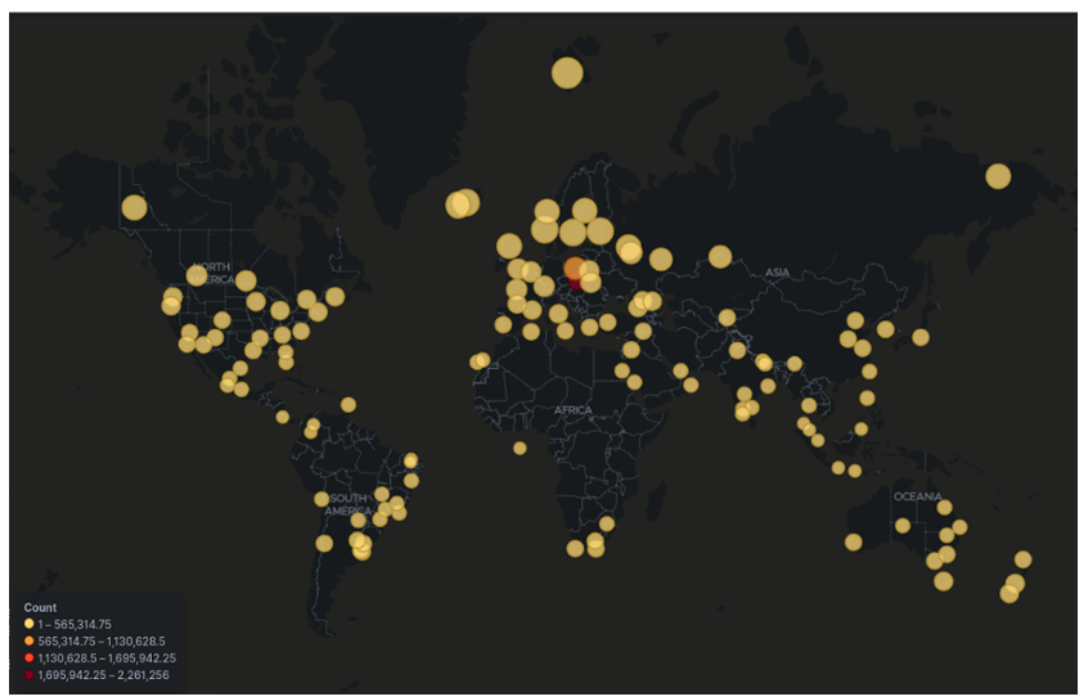

47 institutions / 19 countries / 5 continents / 14200 users / 11300 teams / > 11000000 smartphone detections / > 1200 smartphone work years

Figure 5: Map of the scientific links and activities of the citizen science of the CREDO project (state of art of the project for June 2021)

PAS and other scientific institutions. The number of other people - volunteers supporting the activities of CREDO from time to time (apart from collecting data) is several times greater.

The academics of the CREDO project are open to engaging in scientific discussions as well as on the topics of popularization and didactics. They encourage all teachers to join the "Particle Hunters" program and to involve interested young people in deepening their knowledge of physics and astrophysics, including active participation in the activities of the project.

\section{Acknowledgements}

This research has been supported in part by PLGrid Infrastructure and we warmly thank the staff at ACC Cyfronet AGH-UST for their always helpful supercomputing support. This work was partly financed by a Polish research project with no. 2018/29/B/ST2/02576 (National Science Center) and by the International Visegrad grant No. 21920298.

\section{References}

[1] P. Homola, et al. for the CREDO Collaboration, Cosmic Ray Extremely Distributed Observatory, Symmetry 12, no. 11, (2020) 1835. DOI :10.3390/sym12111835

[2] https://www.facebook.com/107590317754048/videos/2000443506764046

[3] P. Homola, et al. for the CREDO Collaboration, Invitation to the Cosmic Ray Extremely Distributed Observatory, in proceedings of the $37^{\text {th }}$ ICRC 2021, PoS (ICRC2021) 942 


\section{Full Authors List: CREDO Collaboration}

Robert Kamiński ${ }^{1}$, Janusz Firla ${ }^{2}$, Sławomir Stuglik ${ }^{1}$, David Alvarez Castillo ${ }^{1}$, Nikolay Budnev ${ }^{3}$, Olaf Bar ${ }^{4}$, Lukasz Bibrzycki ${ }^{4}$, Alok Chandra Gupta ${ }^{5}$, Bohdan Hnatyk ${ }^{6}$, Piotr Homola ${ }^{1}$, Michał Karbowiak $^{7}$, Marcin Kasztelan ${ }^{8}$, Peter Kovacs ${ }^{9}$, Bartosz Lozowski ${ }^{10}$, Mikhail Medvedev ${ }^{11,12}$, Alona Mozgova ${ }^{6}$, Michał Niedźwiecki $^{13}$, Marcin Piekarczyk ${ }^{4}$, Matias Rosas ${ }^{14}$, Krzysztof Rzecki $^{15}$, Katarzyna Smelcerz ${ }^{13}$, Karel Smolek ${ }^{16}$, Jaroslaw Stasielak ${ }^{1}$, Oleksandr Sushchov ${ }^{1}$, Manana Svanidze ${ }^{17}$, Arman Tursunov ${ }^{18}$, Yuri Verbetsky ${ }^{17}$, Tadeusz Wibig ${ }^{7}$, Jilberto Zamora-Saa ${ }^{19}$

${ }^{1}$ Institute of Nuclear Physics Polish Academy of Sciences, Radzikowskiego 152, 31-342, Kraków, Poland, ${ }^{2}$ CREDO project, ${ }^{3}$ Irkutsk State University, Russia, ${ }^{4}$ Pedagogical University of Cracow, Podchorżych 2, 30-084, Kraków, Poland, ${ }^{5}$ Aryabhatta Research Institue of Observational Sciences (ARIES), Manora Peak, Nainital 263001, India, ${ }^{6}$ Astronomical Observatory of Taras Shevchenko National University of Kyiv, 04053 Kyiv, Ukraine, ${ }^{7}$ University of Lodz, Faculty of Physics and Applied Informatics, Pomorska 149/153, 90-236, Lódź, Poland, ${ }^{8}$ National Centre for Nuclear Research, Andrzeja Soltana 7, 05-400 Otwock-Świerk, Poland, ${ }^{9}$ Wigner Research Centre for Physics, Konkoly-Thege Miklós út 29-33., H-1121, Budapest, Hungary, ${ }^{10}$ Faculty of Natural Sciences, University of Silesia in Katowice, Bankowa 9, 40-007 Katowice, Poland, ${ }^{11}$ Department of Physics and Astronomy, University of Kansas, Lawrence, KS 66045, USA, ${ }^{12}$ Laboratory for Nuclear Science, Massachusetts Institute of Technology, Cambridge, MA 02139, USA, ${ }^{13}$ Department of Computer Science, Faculty of Computer Science and Telecommunications, Cracow University of Technology, Warszawska 24, 31-155 Kraków, Poland, ${ }^{14}$ Liceo 6 Francisco Bauzá, Montevideo, Uruguay, ${ }^{15}$ AGH University of Science and Technology, Mickiewicz Ave., 30-059 Kraków, Poland, ${ }^{16}$ Institute of Experimental and Applied Physics, Czech Technical University in Prague, ${ }^{17}$ E. Andronikashvili Institute of Physics under Tbilisi State University, Georgia, ${ }^{18}$ Research Centre for Theoretical Physics and Astrophysics, Institute of Physics, Silesian University in Opava, Bezručovo nám. 13, CZ-74601 Opava, Czech Republic, ${ }^{19}$ Universidad Andres Bello, Departamento de Ciencias Fisicas, Facultad de Ciencias Exactas, Avenida Republica 498, Santiago, Chile 